\title{
Abstracts for British Paediatric Cardiac Association Annual Meeting: Leicester, 21-22 November 2002
}

\author{
Right ventricular outflow tract reconstruction using Contegra (Venpro) \\ Echocardiographic evaluation and conduits. Short- and mid-term outcome. \\ Single institution experience \\ S. Shebani, Max Bughai, P. Miller, J De Giovanni, J. Wright, O. Stumper, J. Brawn, D. Barron.

\section{Birmingham Children's Hospital, Birmingham, United Kingdom}

Background: Current techniques and strategies for correction of the cardiac anomalies of the RVOT include surgical repair and/or replacement of the pulmonary valve. These techniques include implantation of bioprosthetic conduit consisting of woven polyester tubes as supportive housing for gluteraldehyde-fixed porcine or bovine pericardial valves. Others prefer implantation of a non-valved conduit or Homograft. Contegra Pulmonary Valve Conduit: is a bioprosthesis, which consists of a hetrologous bovine jugular vein, having a trileaflet venous valve and no discontinuity between the lumen of the conduit and the valve it incorporates. In our institution we previously reported our experience with Tissue med and for the last 15 years Homografts and Hancock conduits have been used. Homografts are preferred but because of availability issues and short-term good results of Venpro the latter is being introduced. Objective: To study the natural history of this conduit. Methods: Jan 2000 till June 2002. 49 conduits were placed in RVOT as a definitive procedure. Diagnosis: Tetralogy of Fallot with or with out pulmonary atresia $(\mathrm{PA})(\mathrm{n}=10)$, TOF with LAD arising from RCA $(\mathrm{n}=2)$. PA, VSD MAPCA $(\mathrm{n}=22)$, Truncus arteriosus $(\mathrm{n}=3)$, Aortic atresia, aortic coarctation, VSD $(\mathrm{n}=3)$, TGA, PA, TAPVD, AVSD $(n=1)$, CCTGA $(n=1)$, TGA, VSD, PS $(n=2)$, Conduit replacement $(n=11) 8$ of them were Hancock conduits. Protocol: Detailed cardiac ultrasound: Conduit diameters, PW Doppler, regurgitation fraction, TR RV function, pressures, $\mathrm{dp} / \mathrm{dt}$, propagation over TV in colour M-mode. Studies conducted within 1st months, 3 months, 6 months and one year and in longer FU group 2 years postoperative. Assessment includes CXR, ECG. Patients with abnormal findings and high RV pressures were brought forward for an invasive study. 15/49 FU out side $\mathrm{BCH}$, relevant consultants approached with protocol and results will hopefully be ready by end of October 2002. Initial results: Data is available on 34 pts out of 49: We divided the cohort to 2 groups: initial group Jan 2001-Dec 2001: 31 pts FU (10-34 months) mean 22 months data complete on 23 pts. Late group: (short term FU) Jan 2002-end of June 2002: 17 pts FU period (5-9 months) data complete on 11/17. Results: Death: 6/49 5 unrelated to conduit, one thrombus occlusion of the conduit. 28 alive with complete FU. 2 pts had conduit thrombosis 4-21 days post operation; one died. The second patient developed SBE related to the Venpro conduit required explantation, and replacement with another Venpro conduit, he subsequently developed thrombus on the second conduit valve cusp within 10 days. Thrombophilia screen initiated 2 conduit stenosis one moderate with low RV pressures, second severe with high RV pressures required balloon dilatation. 4 had significant dilatation, 8 had cardiac catheterisation for high RV pressures conduit stenosis or severe dilatation. Conduit dilatation entered into regression analysis against RV pressures with no correlation, on the other hand RV/LV on M-Mode $>60 \%$ seemed to correlate with high RV pressures in 6 cases all secondary to branch pulmonary artery stenosis. Initial conclusion: Good short term results in first 6/12 if no complications within first 4 weeks. Midterm results of 23 pts of initial group 17/23 (73.9\%) had good outcome 6/23 (26.1\%) high RV pressures 1 conduit stenosis 5 branch PA stenosis. 


\title{
Dental preventive experiences of children with congenital heart disease
}

Richard Balmer, Frances Bu'Lock

Glenfield General Hospital, Leicester, United Kingdom

Object: To examine the degree to which children, considered to be at risk from infective endocarditis, had received professional dental health education and preventive procedures and to evaluate parents' knowledge of the link between oral health and infective endocarditis. Materials and methods: 38 children under the care of the department of paediatric cardiology at Glenfield General Hospital, Leicester, participated in the study. Children were aged 2-16 years, had had the cardiac diagnosis made at or around birth and were considered at risk from infective endocarditis. Questionnaires were conducted with the families. Information was gathered about specific types of oral health advice and preventive procedures that they had received from dental professionals. A short dental examination was carried out. Parents were asked if they knew why oral health was of particular importance in their child. Results: $58 \%$ of the children demonstrated evidence of previous or current dental disease. $24 \%$ had at least one filling, $13 \%$ had one or more teeth with an enamel defect and $39 \%$ had untreated dental caries. Only $79 \%$ of children were registered with a dentist. There was no difference in the dental health of registered and non-registered children according to chi squared test. $29 \%$ of the study group had received oral hygiene instruction $(\mathrm{OHI}), 42 \%$ had received dietary advice (Diet), $13 \%$ had received advice regarding fluoride supplementation or had had fluoride professionally applied (F) and $8 \%$ had had fissure sealants (FS). These percentages remained relatively low even if only registered children (OHI $-37 \%$, Diet$53 \%, \mathrm{~F}-17 \%, \mathrm{FS}-10 \%$ ) or only registered children with previous or current dental disease (OHI - 41\%, Diet$59 \%, \mathrm{~F}-12 \%, \mathrm{FS}-12 \%)$ were considered. Only $64 \%$ of parents were aware of the link between the oral health of their children and infective endocarditis. Parents of children who were registered were more likely to be aware of this link, according to chi squared test $(\mathrm{p}<0.05)$, then parents of children who were not registered. Conclusions: In spite of being registered with general dental practitioners, few children with cardiac disease had received basic dental health education. Even children who had experienced dental disease and should, therefore be considered to be more vulnerable, were overlooked. Further education in the importance of maintaining good oral health is required, not just for patients but for doctors and dentists as well. Close links with specialist paediatric dental units which can contribute to the care of these children is essential.

\section{The evolution of effective treatments for the failing Fontan circulation}

\author{
A.T.M. Tang, ${ }^{1}$ K. Roman, ${ }^{2}$ J. Morgan, ${ }^{2}$ A. Salmon, ${ }^{2}$ B. Keeton, ${ }^{2}$ J. Gnanapragasm, ${ }^{2}$ J.V. Vettukattil, ${ }^{2}$ \\ J.L. Monro, ${ }^{1}$ M.P. Haw ${ }^{1}$ \\ ${ }^{1}$ Departments of Cardiology and Cardiac Surgery, Wessex Regional Cardiac and ${ }^{2}$ Thoracic Unit, Southampton General \\ Hospital, Tremona Road, Southampton, United Kingdom
}

Objectives: Many patients with an atriopulmonary Fontan connection develop symptoms related to low cardiac output and/or refractory atrial arrhythmias. We present our experience of converting patients to total cavopulmonary connection (TCPC) with and without arrhythmia surgery. Methods: Between 1997 and 2002 sixteen patients (mean age $19.5 \pm 7.9$ years) underwent conversion surgery $12.3 \pm 3.7$ years after atriopulmonary Fontan. The indication was primarily arrhythmias in 9, obstruction in 4 and poor functional ability in 3 . The preoperative NYHA functional class was II in 2, III in 7 and IV in 7. Four underwent intracardiac lateral tunnel whilst 12 received extracardiac TCPC with right atrial cryoablation. Results: No hospital mortality occurred. One developed conduit obstruction in the immediate postoperative period requiring replacement, another required redo surgery for endocarditis. Average hospitalisation was $18.5 \pm 9$ days; chest drains were removed on day $6 \pm 6$. At follow-up (mean 22 months, range 1-46 months) late atrial arrhythmias had recurred in 2/4 patients with intracardiac TCPC (without ablation) and 1/12 with extracardiac TCPC with ablation. One unexpected late death occurred, and all remaining patients are in NYHA class I or II. Conclusions: Fontan conversion including arrhythmia surgery can be achieved with low mortality, improvement in NYHA class, and reduces hospitalisation. Medium term results suggest that concomitant arrhythmia surgery may reduce the incidence of late arrhythmias. 


\section{Transcatheter closure of multifenestrated atrial septal defects with or without aneurysm using the Helex Septal Occluder}

E. Thomas, B.R. Keeton, J.P. Gnanapragasam, J.J. Vettukattil, A.P. Salmon

The General Hospital, Southampton, United Kingdom

A large number of atrial septal defect occlusion devices have been investigated for percutaneous ASD closure. The Helex Septal Occluder (HSO) ${ }^{\mathrm{TM}}$ is a novel non-self-centering device.

We retrospectively analysed our experience of transcatheter occlusion of multifenestrated ASDs with the HSO at our institution. Five patients with fenestrated ASD underwent HSO implantation over a period of two years from July 2000 to September 2002. Two of these five patients had an associated aneurysmal atrial septum. Amongst these five, three were less than 13 years and two were above 50 years of age.

The size of the devices deployed ranged from 25 to $30 \mathrm{~mm}$. Normal pulmonary artery pressures were noted in all patients and their Qp/Qs ratios ranged from 1.13 to 2.8. Average procedure duration was $94.6 \mathrm{~min}$. Device deployment was satisfactory in all the five patients and there were no complications. The atrial septal aneurysm was well contained and stabilised between the left and right atrial components of the device. Two patients had a tiny residual leak at follow up. We conclude from this experience that the HSO is well suited for closure of multifenestrated atrial septal defects. We found it to be particularly useful in stabilising the atrial septum in those with septal aneurysms and this may be of long-term benefit. 